\title{
A case of celiac disease with neurologic manifestations misdiagnosed as amyotrophic lateral sclerosis
}

\author{
Hyoju Ham, Bo-In Lee, Hyun Jin Oh, Se Hwan Park, Jin Su Kim, Jae Myung Park, Young Seok Cho, \\ Myung-Gyu Choi \\ Department of Internal Medicine, Seoul St. Mary's Hospital, College of Medicine, The Catholic University of Korea, Seoul, Korea
}

Celiac disease (CD) is an immune-mediated enteropathy and is a rare disease in Asia, including in Korea. However, the ingestion of wheat products, which can act as a precipitating factor of $\mathrm{CD}$, has increased rapidly. CD is a common cause of malabsorption, but many patients can present with various atypical manifestations as first presented symptoms, including anemia, osteopenia, infertility, and neurological symptoms. Thus, making a diagnosis is challenging. We report a case of CD that mimicked amyotrophic lateral sclerosis (ALS). The patient was a sexagenary man with a history of progressive motor weakness for 2 years. He was highly suspected as having ALS. During evaluation of his neurological symptoms, esophagogastroduodenoscopy (EGD) was performed because he had experienced loose stools and weight loss for the previous 7 months. On EGD, the duodenal mucosa appeared smooth. A biopsy revealed severe lymphoplasma cell infiltration with flattened villi. His serum endomysial antibody (immunoglobulin A) titer was 1:160 (reference, <1:40). Finally, he was diagnosed as having CD, and a gluten-free diet was immediately begun. At a 4-month follow-up, his weight and the quality of his stool had improved gradually, and the neurological manifestations had not progressed. (Intest Res 2017;15:540-542)

Key Words: Celiac disease; Malabsorption syndromes; Amyotrophic lateral sclerosis

\section{INTRODUCTION}

Celiac disease $(\mathrm{CD})$ is an immune-mediated enteropathy triggered in genetically susceptible individuals by the ingestion of gluten-containing grains. CD occurs in $0.33 \%$ to $0.77 \%$ of individuals in Western countries. ${ }^{1}$ There have been few reports of CD cases in Asia. So far, there has been only 1 report of a suspected case of CD in Korea, ${ }^{2}$ but recently, the consumption of wheat products has increased rapidly, which may be a precipitating factor for CD.

$\mathrm{CD}$ is a common cause of malabsorption, but a large number of individuals have atypical manifestations as their first

Received August 12, 2016. Revised October 5, 2016. Accepted October 14, 2016. Published online March 7, 2017

Correspondence to Bo-In Lee, Department of Internal Medicine, Seoul St. Mary's Hospital, College of Medicine, The Catholic University of Korea, 222 Banpo-daero, Seocho-gu, Seoul 06591, Korea. Tel: +82-2-2258-6024, Fax: +82-2-2258-2055, E-mail: gidoc4u@gmail.com

Financial support: None. Conflict of interest: None. presented symptoms. Extraintestinal manifestations of CD are 15 times more frequent than are intestinal manifestations, making diagnosis extremely challenging. ${ }^{3}$ Herein, we report a case of $\mathrm{CD}$ that mimicked amyotrophic lateral sclerosis (ALS), with neurologic manifestations being the first presented symptoms.

\section{CASE REPORT}

A sexagenary male patient visited our neurology department with progressive motor weakness without sensory manifestations. He had no relevant medical history. When he had visited a foreign hospital for left-sided weakness 2 years earlier, no abnormalities had been found on cerebral and spinal MRI and electromyography. Doxycycline was prescribed for 1 month based on a suspected diagnosis of chronic Lyme disease, as determined by a positive serum IgG Borrelia burgdorferi test result. However, his neurologic manifestations did not improve with treatment.

\footnotetext{
๑ Copyright 2017. Korean Association for the Study of Intestinal Diseases. All rights reserved.

This is an Open Access article distributed under the terms of the Creative Commons Attribution Non-Commercial License (http://creativecommons.org/licenses/by-nc/4.0)

which permits unrestricted non-commercial use, distribution, and reproduction in any medium, provided the original work is properly cited.
} 
Ten months previously, he complained of newly developed weakness in the right leg and both hands. Physical and neurologic examinations revealed muscle atrophy of both extremities, with fasciculation and impaired dorsiflexion in the ankles (Medical Research Council Scale III/II) and toes (II/II), and hyperreflexia in the biceps and brachioradialis. Findings on neurologic examinations of the cranial nerves, cerebellum, and general sensation were normal. Electromyography and nerve conduction studies showed widespread denervation. The physicians were highly suspicious of ALS since the patient's neurologic manifestations progressed gradually, without any sensory disorder, and he had both upper motor neuron signs (including hyperreflexia), and lower motor neuron signs (including muscle atrophy), as well as decreased muscle tone.

When the patient visited our neurology department, laboratory tests, including serum and cerebro-spinal fluid Lyme disease antibody, thyroid function tests, and autoimmune tests were performed to exclude other non-ALS disorders that would cause neurologic manifestations. All the test results were normal, and riluzole was prescribed.

At admission, our gastroenterology department was consulted, because the patient had experienced loose stools and weight loss for the previous 7 months. Stool test results were negative and a glucose hydrogen breath test result was within normal limits. An esophagogastroduodenoscopy was performed; the duodenal mucosa appeared smooth (Fig. 1), and a biopsy revealed severe lymphoplasma cell infiltration with flattened villi (Fig. 2), which is consistent with the duodenal

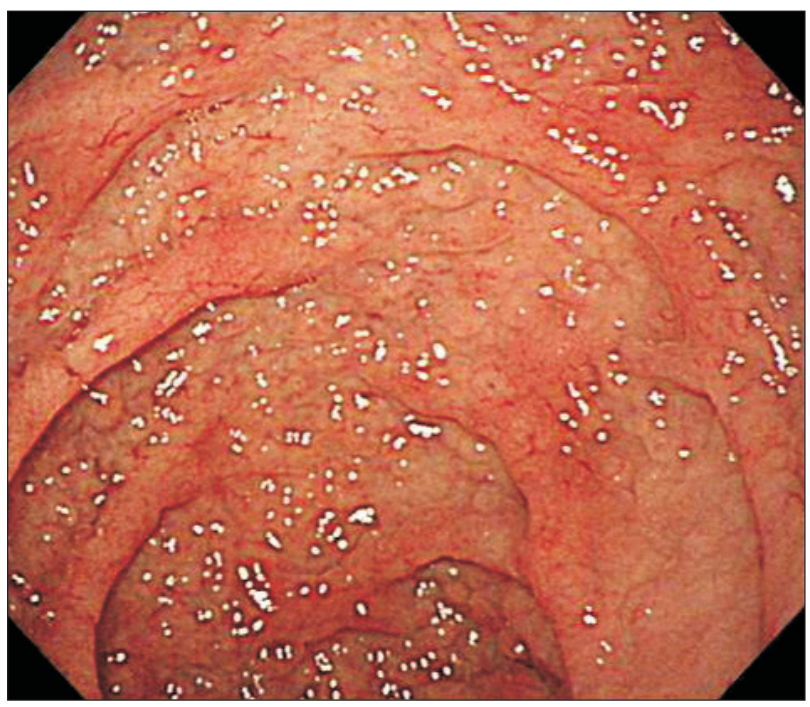

Fig. 1. Esophagogastroduodenoscopy demonstrating characteristic findings of celiac disease. Flattened villi with granularity in the duodenal mucosa. involvement in CD. Capsule endoscopy followed, which showed atrophy of the villi, scalloping, mosaicism, and micronodularity of the proximal small bowel mucosa (Fig. 3). The patient's serum endomysial antibodies (IgA) titer was 1:160 (reference, $<1: 40$ ). An additional medical investigation revealed that he had a cousin with $\mathrm{CD}$. The patient was immediately started on a gluten-free diet. At a 4-month follow-up, his weight had improved gradually, as had the quality of his stools, and the neurologic manifestations had not progressed.

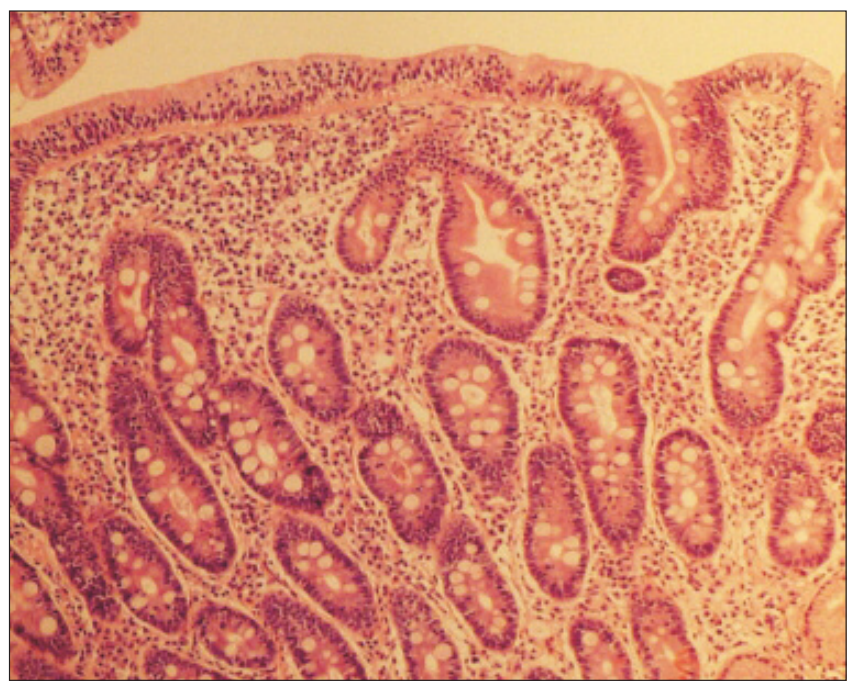

Fig. 2. Duodenal biopsy specimen showing characteristic findings of celiac disease. Flattened villi with Iymphoplasma cell infiltration ( $\mathrm{HCtE}_{\text {, }}$ $\times 100)$.

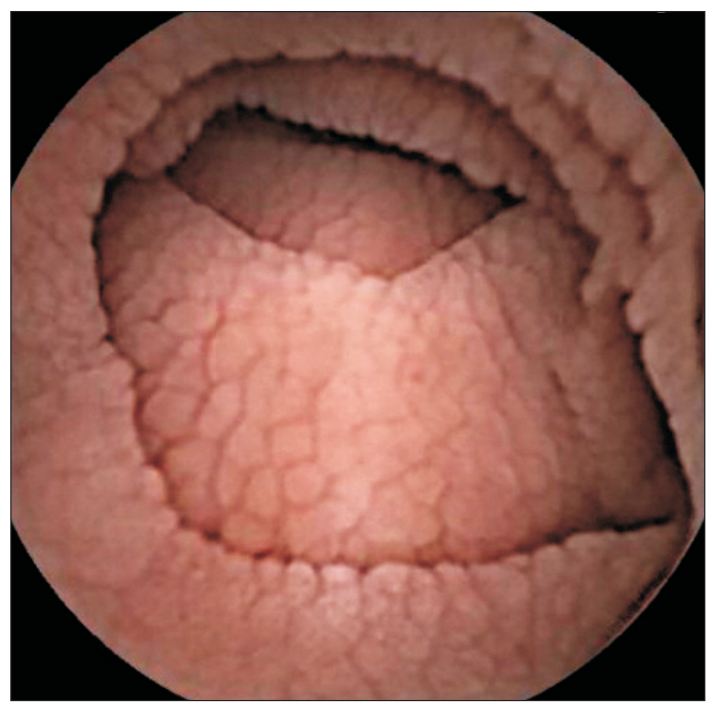

Fig. 3. Capsule endoscopy demonstrating characteristic findings of celiac disease, such as scalloping, a mosaic pattern, and micronodularity with layered folds in the proximal small intestine. 


\section{DISCUSSION}

Diagnosis of CD is challenging because only a small number of patients complain of the classic symptoms related to malabsorption; a much larger number of patients present with atypical manifestations such as anemia, osteopenia, infertility, and neurologic symptoms. ${ }^{4}$ The diagnosis is even more challenging in East Asia, since the prevalence of $\mathrm{CD}$ is extremely low in this area. ${ }^{5}$

In this case, the patient presented with motor weakness, which could easily be misinterpreted as a sign of a neurologic disorder, such as ALS. It is noteworthy that neurologic manifestations such as peripheral neuropathy, myelopathy, cerebellar ataxia, progressive multifocal leukoencephalopathy, and epilepsy are reported in $8 \%$ to $10 \%$ of patients with CD. ${ }^{6}$ Since these neurologic symptoms may be the first manifestation of silent $\mathrm{CD}$, the possibility of $\mathrm{CD}$ should therefore be considered in patients with such symptoms, who cannot be otherwise diagnosed. ${ }^{7}$ If not promptly treated, CD can cause irreversible neurological damage; thus, early diagnosis and treatment is important.

The mechanism of neuronal damage in CD remains unclear, but several hypotheses have been suggested. One is that perivascular inflammation may lead to the breakdown of the blood-brain barrier, allowing an influx of antibodies that cross-react with neural tissue. ${ }^{8}$ Another hypothesis is that malabsorption of vitamins (vitamins B and E), folic acid, and minerals may be associated with neurologic symptoms. However, replacement therapy does not resolve clinical symptoms in the majority of cases, and most patients with $\mathrm{CD}$ that exhibit neurological symptoms do not have nutritional deficiencies. ${ }^{9,10}$ Additionally, a gluten-free diet does not always improve neurologic complications nor prevent the development of neurologic deficits. ${ }^{11}$ Recent investigations have shown that intravenous immunoglobulin therapy may be a suitable treatment for celiac-associated ataxia or multifocal axonal polyneuropathy. ${ }^{12-15}$ For our first patient with $\mathrm{CD}$, the neurologic manifestations had not improved 4 months after the start of a gluten-free diet. However, additional observations are required, since the symptoms, signs, and serologic abnormalities of refractory CD persist for 6 to 12 months after the initiation of a gluten-free diet.

In conclusion, $\mathrm{CD}$ can present with a variety of atypical symptoms, including neurologic manifestations, without gastrointestinal symptoms; consequently, this patient was initially misdiagnosed as having ALS. Therefore, it is important to examine patients carefully in order to avoid missing any clue that could suggest $\mathrm{CD}$. In many cases, improvement in symptoms after implementing a gluten-free diet can help to differentiate $\mathrm{CD}$ from other confusing diagnoses.

\section{REFERENCES}

1. Fasano A, Berti I, Gerarduzzi T, et al. Prevalence of celiac disease in at-risk and not-at-risk groups in the United States: a large multicenter study. Arch Intern Med 2003;163:286-292.

2. Gweon TG, Lim CH, Byeon SW, et al. A case of celiac disease. Korean J Gastroenterol 2013;61:338-342.

3. Fasano A, Catassi C. Current approaches to diagnosis and treatment of celiac disease: an evolving spectrum. Gastroenterology 2001;120:636-651.

4. Kasper DL, Fauci AS, Hauser S, Longo D, Jameson JL, Loscalzo J. Harrison's principles of internal medicine. 2 Volumes. 19th ed. New York: McGraw Hill Education, 2015.

5. Lionetti E, Gatti S, Pulvirenti A, Catassi C. Celiac disease from a global perspective. Best Pract Res Clin Gastroenterol 2015;29:365-379.

6. Cicarelli G, Della Rocca G, Amboni M, et al. Clinical and neurological abnormalities in adult celiac disease. Neurol Sci 2003;24:311-317.

7. Luostarinen L, Pirttilä T, Collin P. Coeliac disease presenting with neurological disorders. Eur Neurol 1999;42:132-135.

8. Hadjivassiliou M, Mäki M, Sanders DS, et al. Autoantibody targeting of brain and intestinal transglutaminase in gluten ataxia. Neurology 2006;66:373-377.

9. Bürk K, Bösch S, Müller CA, et al. Sporadic cerebellar ataxia associated with gluten sensitivity. Brain 2001;124(Pt 5):10131019.

10. Bürk K, Farecki ML, Lamprecht G, et al. Neurological symptoms in patients with biopsy proven celiac disease. Mov Disord 2009;24:2358-2362.

11. Bushara KO. Neurologic presentation of celiac disease. Gastroenterology 2005;128(4 Suppl 1):S92-S97.

12. Bürk K, Melms A, Schulz JB, Dichgans J. Effectiveness of intravenous immunoglobin therapy in cerebellar ataxia associated with gluten sensitivity. Ann Neurol 2001;50:827-828.

13. Chin RL, Tseng VG, Green PH, Sander HW, Brannagan TH 3rd, Latov N. Multifocal axonal polyneuropathy in celiac disease. Neurology 2006;66:1923-1925.

14. Fois A, Vascotto M, Di Bartolo RM, Di Marco V. Celiac disease and epilepsy in pediatric patients. Childs Nerv Syst 1994;10:450454.

15. Nikpour S. Neurological manifestations, diagnosis, and treatment of celiac disease: a comprehensive review. Iran J Neurol 2012;11:59-64. 